\title{
Human Trafficking and HIPAA: What the Health Care Professional Needs to Know
}

\section{Citation}

Powell, Clydette, Michelle Asbill, Samantha Brew, and Hanni Stoklosa. 2017. "Human Trafficking and HIPAA: What the Health Care Professional Needs to Know." Journal of Human Trafficking 4 (2) (September 5): 105-113. doi:10.1080/23322705.2017.1285613.

\section{Published Version}

10.1080/23322705.2017.1285613

\section{Permanent link}

http://nrs.harvard.edu/urn-3:HUL.InstRepos:36304853

\section{Terms of Use}

This article was downloaded from Harvard University's DASH repository, and is made available under the terms and conditions applicable to Other Posted Material, as set forth at http:// nrs.harvard.edu/urn-3:HUL.InstRepos:dash.current.terms-of-use\#LAA

\section{Share Your Story}

The Harvard community has made this article openly available.

Please share how this access benefits you. Submit a story.

Accessibility 


\section{Journal of Human Trafficking \\ Human Trafficking and HIPAA: What the health care professional needs to know --Manuscript Draft--}

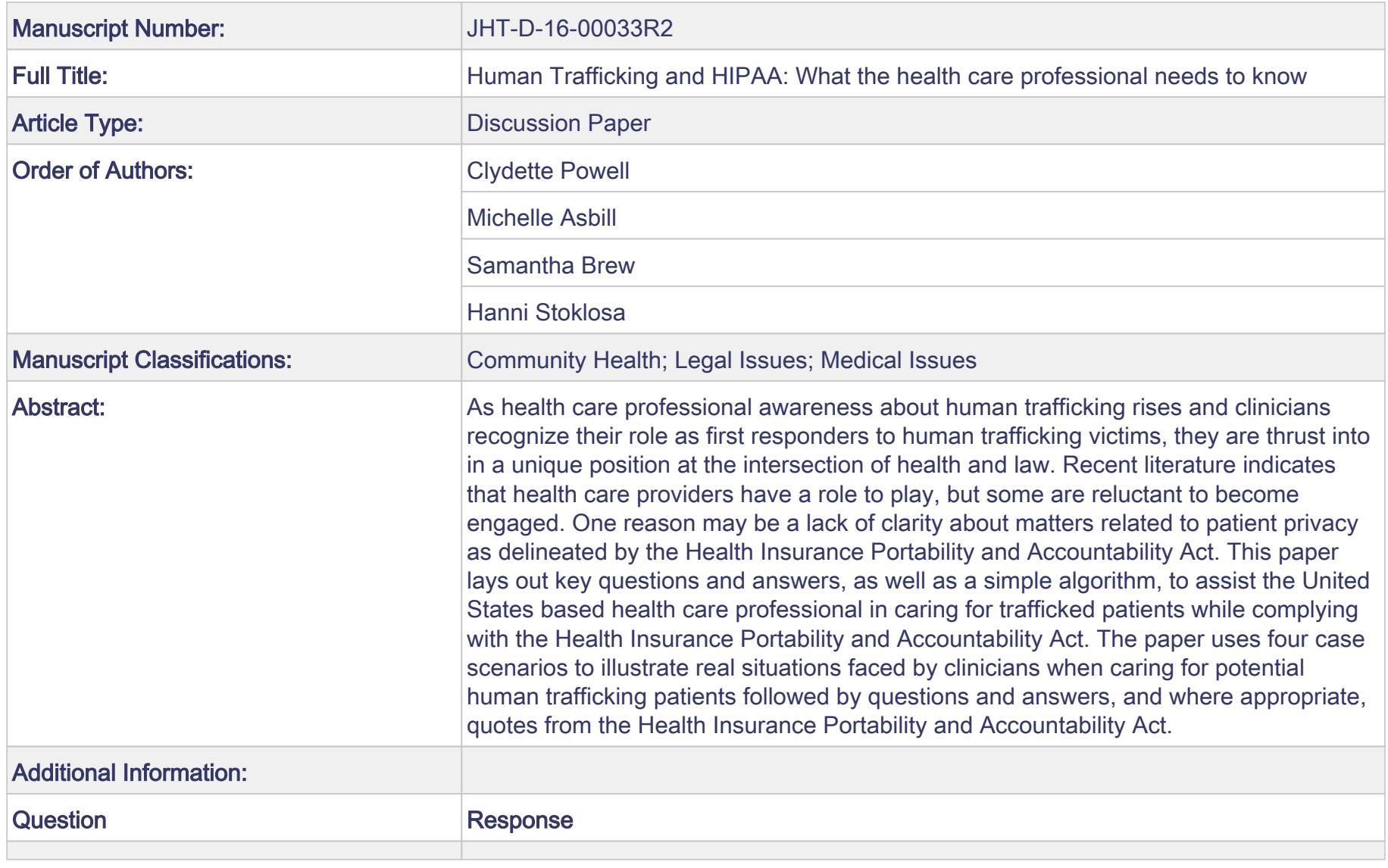


Human Trafficking and HIPAA: What the health care professional needs to know

Clydette Powell, MD, MPH, FAAP [The George Washington University School of Medicine and Health Sciences]

Michelle Asbill, M.S.W. [University of Illinois Urbana-Champaign]

Samantha Brew, [George Washington University]

Hanni Stoklosa*, MD, MPH [Harvard Medical School, Brigham and Women's Hospital, Boston MA]

*Corresponding author: 75 Francis St, Boston MA 02115 hstoklosa@partners.org 
1

Trafficking and HIPAA

Acronyms:

CPS Child Protective Services

DHHS Department of Health and Human Services

ED emergency department

EMS Emergency Medical Services

HCP health care professional

HIPAA Health Insurance Portability and Accountability Act

HT human trafficking

LE law enforcement

NHTH National Human Trafficking Hotline

PHI protected health information

US United States 
Trafficking and HIPAA

\section{Introduction}

As health care professionals (HCPs) become increasingly aware of human trafficking (HT) and recognize their role as first responders, they are sometimes thrust into a position at the intersection of health and law. HCPs who work in emergency departments (ED), urgent care centers, reproductive health clinics, and community health centers may be the first to encounter a patient whom they suspect of being trafficked (Chang, Lee, Park, Sy, \& Quach, 2015; ChisolmStraker et al., 2016; Gibbons \& Stoklosa, 2016; Lederer, \& Wetzel, 2014;). Recent literature indicates that HCPs have a role to play, but some are reluctant to become engaged (Beck et al., 2015; Becker \& Bechtel, 2015; CdeBaca \& Sigmon, 2014; Grace, Ahn, \& Konstantopoulos, 2014; Grace et al., 2014). One reason may be a lack of clarity about matters related to patient privacy as delineated by the Health Insurance Portability and Accountability Act (HIPAA).

This paper lays out key questions and answers, as well as a simple algorithm, to assist the United States (US) based HCP in caring for trafficked patients while complying with HIPAA. ${ }^{1}$ Specifically, it is written for HCPs who are recognized as "covered entities" by HIPAA (Centers for Medicare and Medicaid Services, 2013). We recognize that state and federal laws change over time. This article is not meant to substitute for formal legal advice. Furthermore, we limit our discussion of mandated reporting to its relationship with HIPAA. To our knowledge, this is the first paper to address HIPAA privacy concerns in relation to HCP referral and reporting on suspected cases of HT.

\section{Case Scenarios}

\footnotetext{
${ }^{1}$ As experts in health and human trafficking, we consulted with medico-legal HT experts to confirm that our application and framing of HIPAA was accurate.
} 


\section{Trafficking and HIPAA}

The following scenarios illustrate four different potential human trafficking clinical encounters. At the end of this paper, the cases are discussed briefly in the context of HIPAA and suggested responses are presented.

\section{Case 1: Mandatory reporting}

A 15 -year old female is brought to a community health center by her parents for evaluation of pelvic pain. Human trafficking of minors falls under mandated reporting requirements for HCPs in this state. The HCP interviews her without her parents present. The HCP first discusses the limits of confidentiality with the patient before starting a conversation that might lead to a disclosure. The patient eventually discloses that she has had more than 20 sexual partners, typically does not use condoms, and has a change in her vaginal discharge. Upon further discussion, she shares that, for the last three months, she has been forced by a gang to engage in sex acts at a nearby motel every Friday night. Her parents have no idea and think she just stays out late on weekends. The patient tests positive for gonorrhea and chlamydia. The patient does NOT want her exploitation reported since her parents would be mortified and the gang may retaliate.

\section{Case 2: Disclosing protected health information (PHI) with permission}

A 25-year old female is brought into the ED by her cousin and has a deep laceration on her left palm. The patient is originally from Guatemala and has a limited vocabulary in English. She is living with her cousin here in the US while applying for US citizenship. Additional history obtained from the cousin reveals that the laceration occurred while the patient was preparing dinner. The nurse notices bruising on the upper arms and recognizes the need to interview the patient alone about possible physical abuse and/or labor trafficking. Ultimately, the patient discloses that she is in a dangerous situation, which includes elements of labor and sex 
Trafficking and HIPAA

trafficking and asks for help to leave her situation. She agrees to allow the HCP to speak with community-based service providers outside the hospital in order to coordinate her care.

Case 3: Need for guidance in caring for a potential victim of HT

A 34-year old male presents to the ED with a chief complaint of arm pain. He speaks

Cape Verdean creole primarily. On history, and states that he fell down the stairs but is reluctant to provide further details. He is accompanied by a female who offers to translate. On musculoskeletal exam, his left upper arm appears deformed. On skin exam, he has bruises in multiples stages of healing. The HCP is concerned that there may be physical abuse and/or human trafficking, however because the health facility has no HT protocol in place it is unclear what next steps to take, including what screening questions to ask.

\section{Case 4: Disclosing PHI without consent, due to imminent threat to safety}

A 19-year old male is brought in by ambulance at 3 am by Emergency Medical Services (EMS). The EMS provider states that the patient called 911 because he was being threatened. The paramedic also states that a vehicle followed the ambulance to the hospital. On exam, the patient is confused, and seems to be under the influence of a substance. He is dressed in tight fitting clothing. He shares that "Derek" said he was going to put a "crack in his skull" because he did not bring home enough money last night from a "date." Hospital security personnel alert the HCP to the fact that there is a man who keeps coming to the front of the ED inquiring about the patient's whereabouts and making threats. Security is visibly fearful of this man.

\section{HIPAA Overview}

The following section provides, in a question-and-answer format, some of the HIPAA basics, followed by the resolution of the case scenarios presented.

Question: What is HIPAA? 
Trafficking and HIPAA

Answer: According to the guide from the US Department of Health and Human Services (DHHS), HIPAA, implemented in 1996, introduced the "Privacy Rule" as a means to provide protection and standards for the use and release of patient information (DHHS, n.d.(a)). The goal was to design a flexible policy framework that would guard patient information, but allow and define specific instances and procedures where disclosure would be necessary (DHHS, n.d.(a)). Question: Which entities must comply with HIPAA?

Answer: HIPAA uses the term "covered entities" to describe who must comply with HIPAA. "Covered entities" include most HCPs (referred to as "health care providers" in the HIPAA policy), health plans and "healthcare clearinghouses" (such as billing or management companies using health information). The Centers for Medicare and Medicaid (2013) provide an online tool for determining covered entity status. In addition, subsequent to passage of the Health Information Technology for Enforcement and Clinical Health Act of 2009, "business associates”, which provide services to or for the benefit of covered entities, are also directly subject to HIPAA compliance and reporting requirements with respect to the PHI they come into contact with when providing those services. If a HCP is not a HIPAA covered entity or the business associate of a covered entity, the HCP is not subject to HIPAA in relation to disclosures of PHI. Question: What is protected health information (PHI)?

Answer: HIPAA defines PHI as "individually identifiable health information," which "identifies the individual or for which there is a reasonable basis to believe can be used to identify the individual" (name, birthdate) and includes certain demographic information concerning medical conditions (past, present, and future), the dates of medical care provision, and information related to the payment of rendered care (DHHS, n.d. (a), p. 3; DHHS, 2013a). Question: When is a PHI disclosure permitted? 
Trafficking and HIPAA

Answer: HIPAA instructs that a disclosure of PHI is only permitted

a) when the HCP receives permission from the individuals or their personal representative (as defined by HIPAA) or

b) in circumstances specifically defined by HIPAA that require or allow for disclosure, each of which is further defined and explained by HIPAA (DHHS, n.d. (a), p. 4-5).

Below, we elaborate on these permitted disclosures as they relate to HT and HIPAA.

\section{HIPAA Operationalized}

Question: Is a HCP, covered by HIPAA, permitted to disclose PHI in the case of a suspected HT victim?

Answer: Yes, the HCP, covered by HIPAA, is permitted to disclose PHI in the case of a suspected HT victim under the following conditions:

a) with the patient's permission or

b) if the patient does not give permission, then reporting is only permitted under specific exclusions or exemptions defined by HIPAA:

1) Mandated reporting: HIPAA allows reporting if state law specifically mandates

a disclosure for suspected trafficking of a minor, such as in Colorado or

Massachusetts (see mandatory reporting question) or where HT falls under child abuse mandated reporting laws (Atkinson, Curnin, \& Hanson, 2016). When such reporting is mandated, the reporting must be made only to the legal entity authorized to receive these reports, and should include a disclosure of only the information required under mandated reporting laws.

2) Imminent danger of patient or staff: The HCP is directed to make decisions in "good faith" and may disclose PHI when "it is felt to be necessary to prevent or 


\section{Trafficking and HIPAA}

lessen a serious and imminent threat to the health or safety of a person or the public" (DHHS, 2013b).

Question: When should HCPs get consent from patients when disclosing PHI?

Answer: If disclosure of PHI is not mandated by law or otherwise permitted by HIPAA as described above, the HCP must ask the patient for consent to disclose PHI. This is true if the HCP is disclosing PHI when speaking with anti-HT service providers or trying to access legal, housing, and law enforcement (LE) resources for the patient, such as calling the National Human Trafficking Hotline (NHTH). The consent is ideally in the form of written authorization, but may be in verbal form, and should be documented in the medical record (DHHS, n.d. (b)).

\section{HIPAA and Mandated Reporting}

Question: Under what kinds of "mandatory reporting" state laws does HT fall?

Answer: Some state laws specifically require HCPs to report suspected cases of HT of minors, but all fifty states and the District of Columbia have mandatory reporting laws requiring HCPs to report child abuse and neglect. However, in a limited number of states, under certain circumstances, the HT of minors may not be considered a form of child abuse and trafficking of minors may not be reportable (Atkinson, Curnin, \& Hanson, 2016). Shared Hope International provides a list indicating which states have sexual exploitation in their child abuse and neglect laws (Shared Hope International, 2014). Conversely, in the state of Massachusetts, for example, HT of children is explicitly mentioned as a mandated reporting requirement under child abuse statutes (189th General Court of the Commonwealth of Massachusetts, n.d.). Of note, mandated reporting laws are controversial, and some National Academy of Medicine experts caution that "adopting a universal reporting requirement without ensuring the adequate preparation of child 
Trafficking and HIPAA

welfare agencies may have unintended consequences that are harmful to the vulnerable children that the laws are designed to assist" (2013, p. 182).

\section{Case Resolutions}

\section{General Principles}

When a HCP suspects a patient is a HT victim, the core of the approach should be patient-centered and trauma-informed. A trauma-informed interviewer and a professional interpreter, when English is not the first language, should be drawn into the process if at all possible, and the patient interviewed alone. The patient should be informed of the limits of confidentiality prior to the interview. Multidisciplinary care for a victim of HT should be guided by the patient's stated needs, state laws, and will likely take many forms, including medical and mental health care, connection with shelter, substance use disorder treatment, etc. (see Figure 1 for possible paths). Each of the cases below illustrates the importance of having a healthcare system wide response protocol in place that standardizes and tailors this care (Baldwin, Barrows, Stoklosa, 2017; Chang et al., 2015; Stoklosa, Dawson, Williams-Oni, \& Rothman, 2016; Stoklosa, Showalter, Melnick, \& Rothman, 2016) (see Figure 2).

\section{Case 1: Mandatory reporting}

This case takes place in a US state where human trafficking of those under the age of 18 falls under mandated reporting requirements. It is implied that the parents will find out. It is recommended that the HCP talk at length with the patient to explain what needs to be done, and volunteer to help her tell her parents (e.g., offer to be in the room, and even offer to tell the parents, if that is what she wants). Moreover, the HCP should have a long discussion with the parents, emphasizing the child's victim status (she is not to blame) and the need for the parents to 
Trafficking and HIPAA

support and protect her. The HCP should ask if the patient wants to be present when the authorities are called, so she can tell them what she thinks they need to know.

Depending on state law, reporting may be made to Child Protective Services (CPS)

and/or LE. According to HIPAA, the report should include a disclosure of only the information required under mandated reporting laws. Note, it is important, where possible, to work with CPS and LE staff that have been trained specifically on trafficking because of the unique needs of this population and the potential for unintended harm to the patient by untrained CPS or LE staff. The National Human Trafficking Hotline (NHTH) may be helpful in identifying trained CPS or LE staff. Through grants from the US DHHS, the 24/7/365 hotline (1-888-373-7888), is available in over 200 languages, for any caller who needs information and guidance on matters concerning HT. Because of the concerns for safety related to potential gang retaliation, regardless of mandated reporting, referral to LE may be considered as a part of safety planning. The HCP should emphasize to LE the child's fear of gang retaliation and the special need for safety measures.

The question arises about what to do in the rare circumstance when caring for a child who is being trafficked and the state does NOT require mandatory reporting. In these scenarios, just as in caring for adult victims of trafficking, a clinician should connect the patient with the multidisciplinary resources that best match his/her stated needs and are most likely to improve his/her safety and health.

\section{Case 2: Disclosing PHI with permission}

This patient's story raises concerns for trafficking, signals the patient's request for help, and illustrates how protected health information (PHI) can be disclosed with the patient's permission. The consent is ideally in the form of written authorization, but may be in verbal 
Trafficking and HIPAA

form, and should be documented in the medical record (DHHS, n.d. (b)). Depending on the patient's goals and existing HT health system protocols, the response may first include utilizing social workers and/or contacting the National Human Trafficking Hotline (NHTH) in order to identify local non-governmental organizations that could provide resources such as housing, substance use disorder, or legal services. The ideal is for the patient to speak with NHTH directly so that the case can be discussed in detail. If the HCP calls the NHTH, the hotline can provide technical assistance including accessing community HT resources. If the HCP contacts the NHTH and has consent from the patient to share PHI, he/she may discuss the case in detail with the NHTH. When calling NHTH without explicit consent from the patient, it is important to share only non-PHI, possibly using hypothetical questions such as: "If I were caring for a male patient from the US who may be labor trafficked, what are local legal services that I can access? Are there any shelter options?" Note, in this example, the HCP mentions the type of trafficking and gender but does not disclose the date of service.

\section{Case 3: Need for guidance in caring for a potential victim of HT}

This case raises the HCP's index of suspicion for trafficking but she needs further guidance on how to explore the possibility of HT with the patient as her facility does not have a protocol in place. As stated previously, it is essential that a trauma-informed interviewer be present, that the patient be interviewed alone without the person who accompanied him/her, and that a professional interpreter be used when the patient's first language is not English. The HCP may choose to access her facility's multidisciplinary resources such as social workers or external resources such as the National Human Trafficking Hotline (NHTH) to gain guidance on questions to ask. The NHTH call specialists are trained to speak with health care providers, and 
Trafficking and HIPAA

walk them through potential screening questions. (See Case 2 discussion for details about engaging NHTH with and without patient consent.) Importantly, the ultimate goal of a clinical encounter like this is not disclosure or rescue. If, after further exploration, the patient does not disclose human trafficking, the HCP should still consider providing safety planning and anticipatory guidance to the patient as they may be at high risk for trafficking. As a safety line, the NHTH number can always be discreetly given to a potential victim, for him/her to call or text at a later date (Call: 1-888-373-7888; Text: HELP to BeFree (233733)). In addition, a follow-up appointment may be made with the patient to return. Overall, it should be clear that the health care setting is a safe haven for the potential victim to return.

\section{Case 4: Disclosing PHI without consent, due to imminent threat to safety}

This case raises the issue of imminent danger. As the patient is fearful himself, he might consent for LE involvement, so he should be asked. However, in this case, there is also perceived imminent danger to the patient and to the staff, and calling LE without the patient's consent is permitted by HIPAA. It is important to understand that trafficking survivors may have prior warrants or charges related to their trafficking exploitation, and so involving LE must be done carefully with LE who have HT training. A minimal amount of information should be disclosed. [place Figure 1 here]

[place Figure 2 here]

\section{Conclusion and Recommendations}

A health care setting that is informed and equipped on the intersections of human trafficking (HT), HIPAA, and mandatory reporting will be better able to serve suspected HT 
Trafficking and HIPAA

victims. The following general recommendations are made in order to guide HCPs in caring for trafficked persons:

- Foremost, and in conjunction with the consulted authorities, health systems should consider the development of a HT protocol which incorporates guidance on HIPAA and mandatory reporting laws (Baldwin, Barrows, Stoklosa, 2017; Stoklosa, et al., 2016). (Figure 2);

- HCPs may use the NHTH hotline to be guided through a trafficking screening interview and identify services for trafficked persons, particularly when a protocol is not in place; and

- HCPs should familiarize themselves with their state's mandated reporting laws applicable to human trafficking.

Acknowledgements: The authors would like to give a special thanks for the perspectives provided by Rochelle Rollins, Katherine Chon, Jordan Greenbaum, Jeffrey Barrows, Holly Atkinson, Nicole Moler, Maralee Gutierrez, Mariam Garuba, and Rachel DiSanto. The authors would also like to thank the US Department of State's Virtual Student Foreign Service program for its support in the recruitment of student interns for participation in this project. 
Trafficking and HIPAA

\section{References}

Atkinson, H., Curnin, K., \& Hanson, N. (2016). U.S. State Laws Addressing Human Trafficking: Education of and Mandatory Reporting by Health Care Providers and other Professionals. Journal of Human Trafficking, 2(2), 111-138.

Baldwin, S., Barrows, J., Stoklosa, H. (2017). Protocol Toolkit for Developing a Response to Victims of Human Trafficking in Health Care Settings. HEAL Trafficking and Hope for Justice. Retrieved from http://www.HEALtrafficking.org.

Beck, M. E., Lineer, M. M., Melzer-Lange, M., Simpson, P., Nugent, M., \& Rabbitt, A. (2015). Medical Providers' Understanding of Sex Trafficking and Their Experience with At-Risk Patients. Pediatrics, (Journal Article). http://doi.org/10.1542/peds.2014-2814

Becker, H. J., \& Bechtel, K. (2015). Recognizing victims of human trafficking in the pediatric emergency department. Pediatric Emergency Care, 31(2), 144-147.

CdeBaca, L., \& Sigmon, J. N. (2014). Combating trafficking in persons: a call to action for global health professionals. Global Health, Science and Practice, 2(3), 261-267.

Chang, K. S., Lee, K., Park, T., Sy, E., \& Quach, T. (2015). Using a clinic-based screening tool for primary care providers to identify commercially sexually exploited children. Journal of Applied Research on Children: Informing Policy for Children at Risk, 6(1), 6.

Centers for Medicare and Medicaid Services. (2013). Are You a Covered Entity? Retrieved from https://www.cms.gov/Regulations-and-Guidance/HIPAA-AdministrativeSimplification/HIPAAGenInfo/AreYouaCoveredEntity.html 
Trafficking and HIPAA

Chisolm-Straker, M., Baldwin, S., Gaïgbé-Togbé, B., Ndukwe, N., Johnson, P. N., \&

Richardson, L. D. (2016). Health care and human trafficking: we are seeing the unseen. Journal of health care for the poor and underserved, 27(3), 1220-1233.

Gibbons, P., \& Stoklosa, H. (2016). Identification and treatment of human trafficking victims in the emergency department: a case report. The Journal of emergency medicine, 50(5), 715-719.

Grace, A. M., Ahn, R., \& Konstantopoulos, W. M. (2014). Integrating curricula on human trafficking into medical education and residency training. JAMA Pediatrics, 168(9), 793794.

Grace, A. M., Lippert, S., Collins, K., Pineda, N., Tolani, A., Walker, R., ... Horwitz, S. M. (2014). Educating health care professionals on human trafficking. Pediatric Emergency Care, 30(12), 856-861.

IOM (Institute of Medicine) and NRC (National Research Council). (2013). Confronting commercial sexual exploitation and sex trafficking of minors in the United States. Washington, DC: The National Academies Press. Retrieved from http://www.ojjdp.gov/pubs/243838.pdf

Lederer, L., Wetzel, C. A. (2014). The Health Consequences of Sex Trafficking and Their Implications for Identifying Victims in Healthcare Facilities. Annals of Health Law, 23(1), 61-91.

Shared Hope International. (2014). State Law Survey: Factors Affecting Child Protective Services Involvement in Commercial Sexual Exploitation of Minors Cases. Retrieved from: http://sharedhope.org/wp-content/uploads/2014/11/State-Law-Survey-2014- 
Trafficking and HIPAA

Likelihood-of-CPS-Involvement-Shared-Hope-International.pdf Accessed June 16, 2015.

Stoklosa, H., Dawson, M. B., Williams-Oni, F., \& Rothman, E. F. (2016). A Review of US Health Care Institution Protocols for the Identification and Treatment of Victims of Human Trafficking. Journal of Human Trafficking, 1-9.

Stoklosa, H., Showalter, E., Melnick, A., \& Rothman, E. F. (2016). Health Care Providers' Experience with a Protocol for the Identification, Treatment, and Referral of HumanTrafficking Victims. Journal of Human Trafficking, 1-11.

The 189th General Court of the Commonwealth of Massachusetts. (n.d.). Reporting of suspected abuse or neglect; mandated reporters; collection of physical evidence; penalties; content of reports; liability; privileged communication, (Laws/Statutes), 51A.

U.S. Department of Health and Human Services. (n.d.) (a). Summary of the HIPAA privacy reporting law. Retrieved from http://www.hhs.gov/ocr/privacy/hipaa/understanding/summary/index.html.

U.S. Department of Health and Human Services. (n.d.) (b). OCR Privacy Brief: Summary of the HIPAA Privacy Rule. Retrieved from http://www.hhs.gov/sites/default/files/privacysummary.pdf

U.S. Department of Health and Human Services Office for Civil Rights. (2013a). HIPAA Administrative Simplification: Regulation Text: 45 CFR Parts 160, 162, and 164: 45 C.F.R. $§ 160.103$ (Unofficial Version, as amended through March 26, 2013). Retrieved from http://www.hhs.gov/sites/default/files/hipaa-simplification-201303.pdf 
Trafficking and HIPAA

U.S. Department of Health and Human Services Office for Civil Rights. (2013b). HIPAA

Administrative Simplification: Regulation Text: 45 CFR Parts 160, 162, and 164: 45 CFR 164.512(j) Uses and disclosures to avert a serious threat to health or safety. (Unofficial Version, as amended through March 26, 2013). Retrieved from

http://www.hhs.gov/sites/default/files/hipaa-simplification-201303.pdf 
Figure 1

Following HIPAA and caring for the trafficked patient, using the National Human Trafficking Hotline (NHTH)

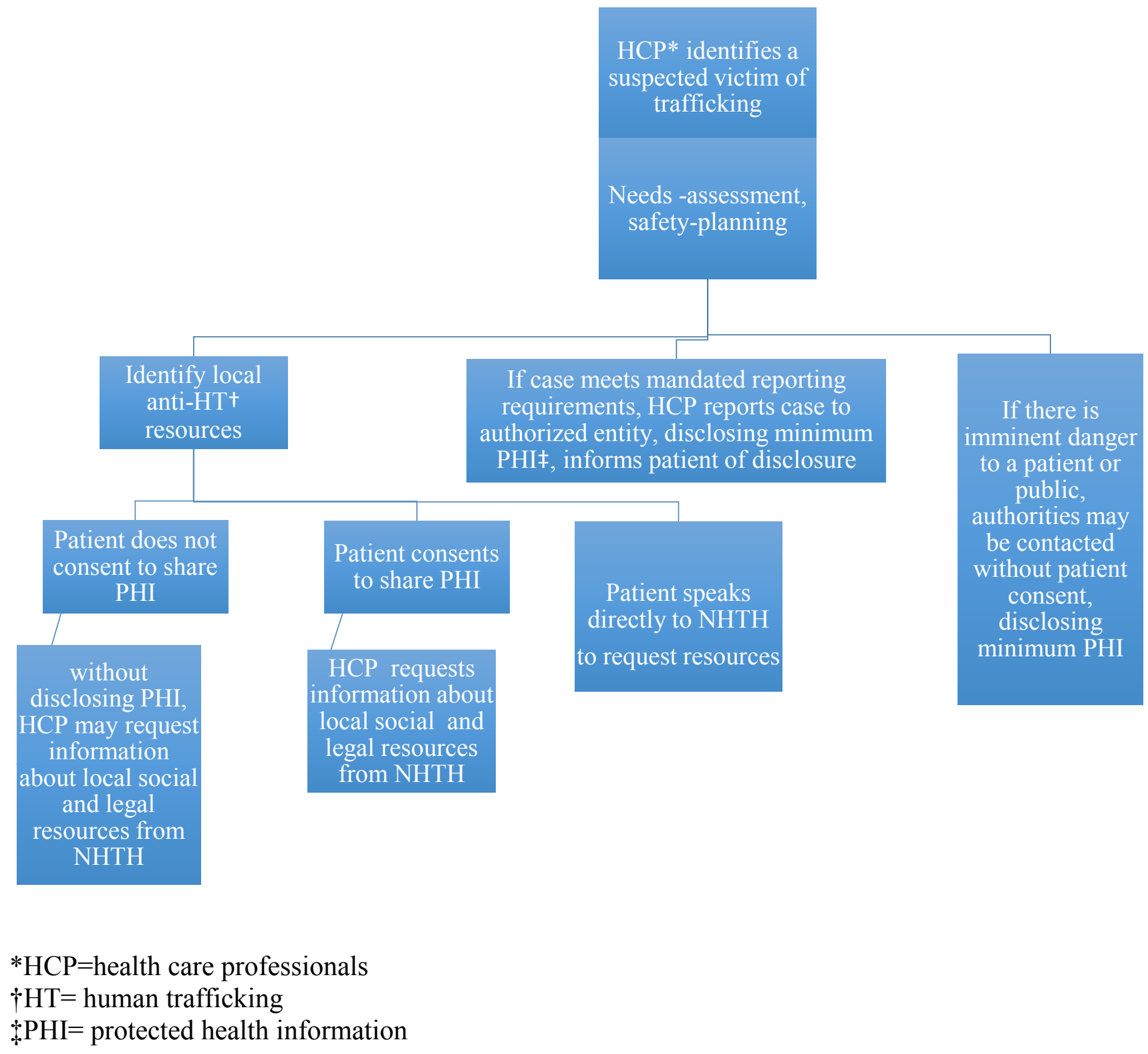




\section{Figure 2}

Considerations for health care institutions in designing a human trafficking (HT) protocol

\section{Step 1: Design HT protocol for healthcare setting:}

A) Meet with institution's privacy officer to learn your institution's policy for PHI, HIPAA, and relevant reporting procedures and documentation germane to HT victims.

B) Know your state's mandatory reporting requirements. Familiarize yourself with your local HT-trained LE*, CPS ${ }^{\text {, }}$, and the other authorities to whom you may be required to report.

C) Understand the services available to potential victims of trafficking in your area, including shelter, medical care, substance use disorder treatment, and mental health services.

D) Organize team of multidisciplinary healthcare and community stakeholders

E) Meet with administration and stakeholders to create protocol, and harmonize protocol with existing protocols for other forms of interpersonal violence, including child abuse, sexual assault, and domestic violence.

Step 2: Train your staff on human trafficking, trauma-informed care, and the protocol; consider simulation cases as part of an interactive training.

Step 3: Display posters or public information that will encourage HCP awareness of HT, and presence of HT protocol.

Step 4: Evaluate and modify the protocol based on data collected, patient outcomes, stakeholder feedback \& changes in laws and policies.

* LE=law enforcement 
$\uparrow \mathrm{CPS}=$ child protective services

the cautious about posting the information in an area where a potential trafficker may view it 